\title{
7he MHC Class II Transactivator (CIITA): A Physiologic Inhibitor of HTLV-2 Retroviral Infection
}

\author{
Giovanna Tosi ${ }^{1}$, Elisabetta Pilotti ${ }^{2}$, Andrea De Lerma Barbaro1, \\ Lorenzo Mortara ${ }^{1}$, Claudio Casoli ${ }^{2}$ and Roberto S Accolla* ${ }^{*}$
}

\author{
Address: ${ }^{1}$ Department of Clinical and Biological Sciences, University of Insubria, 21100 Varese, Italy and ${ }^{2}$ Department of Clinical Medicine, \\ Nephrology, and Prevention, University of Parma, Parma, Italy \\ Email: Roberto S Accolla* - roberto.accolla@uninsubria.it \\ * Corresponding author $\ddagger$ Presenting author
}

from 2005 International Meeting of The Institute of Human Virology

Baltimore, USA, 29 August - 2 September 2005

Published: 8 December 2005

Retrovirology 2005, 2(Suppl I):P5 doi:I0.II86/I742-4690-2-SI-P5

The transcriptional activator CIITA is the master regulator of the expression of MHC class II genes. In addition to this major role, we have found that CIITA exerts an important inhibitory effect on the HTLV-2 replication, similar to our previously decribed effect on the HIV-1 replication. This inhibition is mediated by the N-terminal $1-321$ region where we identified a minimal fragment of 80 aminoacids that specifically blocks the activity of the viral transactivator Tax2. This fragment does not inhibit the function of Tat, the transcriptional activator of HIV-1.

To unveil the biochemical basis of the CIITA-mediated inhibition of Tax 2 we first focussed on the identification of the cellular cofactors used by Tax2 to transactivate the viral promoter. DNA-binding factors (CREB, NFYB) and several co-activators involved in chromatin remodeling (CBP, p300, PCAF, BRG1) are known to interact with or stimulate the transcriptional activity of both CIITA and Tax1, the HTLV-1 homologous of Tax2. Preliminary data indicate that the transactivation activity of Tax 1 and Tax2 is differently influenced by the hystone acetyltransferases CBP, p300 and PCAF providing new informations on the biology of HTLV-2. Furthermore, none of these factors was able to reverse the inhibitory action of CIITA on Tax2 function. Interestingly, we found that the $B$ and, to a lesser extent, the A subunits of the NFY complex inhibit Tax2 activity when iper-expressed in cells. On the basis of our results and of the reported physical interactions between NFY and both CIITA and Tax1, we propose a molecular model for the CIITA-mediated inhibition of Tax 2 activity via the binding of the CIITA-NFY complex to Tax2. When expressed in cells CIITA interacts with the NFY complex; this interaction changes the conformation of NFY increasing its binding affinity for Tax2. Following this model the inhibition of Tax2 by CIITA it is not due to the squelching of a transcriptional positive co-activator, but instead to the recruitment of a cellular factor, NFY, with a negative regulatory action on Tax2. This as well as possible alternative models are presently under scrutiny.

On the whole these results confirm that CIITA may represent a physiologic tool for novel therapeutic strategies aimed at counteracting HLTV-2 replication and spreading. 